\title{
A!
}

This is an electronic reprint of the original article.

This reprint may differ from the original in pagination and typographic detail.

Pulkki, Ville; Lähivaara, Timo; Huhtakallio, Ilkka

\section{Effects of flow gradients on directional radiation of human voice}

Published in:

Journal of the Acoustical Society of America

DOI:

$10.1121 / 1.5025063$

Published: 01/02/2018

Document Version

Publisher's PDF, also known as Version of record

Please cite the original version:

Pulkki, V., Lähivaara, T., \& Huhtakallio, I. (2018). Effects of flow gradients on directional radiation of human voice. Journal of the Acoustical Society of America, 143(2), 1173-1181. https://doi.org/10.1121/1.5025063

This material is protected by copyright and other intellectual property rights, and duplication or sale of all or part of any of the repository collections is not permitted, except that material may be duplicated by you for your research use or educational purposes in electronic or print form. You must obtain permission for any other use. Electronic or print copies may not be offered, whether for sale or otherwise to anyone who is not an authorised user. 


\section{Effects of flow gradients on directional radiation of human voice}

Ville Pulkki, Timo Lähivaara, and Ilkka Huhtakallio

Citation: The Journal of the Acoustical Society of America 143, 1173 (2018); doi: 10.1121/1.5025063

View online: https://doi.org/10.1121/1.5025063

View Table of Contents: http://asa.scitation.org/toc/jas/143/2

Published by the Acoustical Society of America

\section{Articles you may be interested in}

Cancellation of room reflections over an extended area using Ambisonics

The Journal of the Acoustical Society of America 143, 811 (2018); 10.1121/1.5023326

Passive, broadband suppression of radiation of low-frequency sound

The Journal of the Acoustical Society of America 143, EL67 (2018); 10.1121/1.5022192

Reverberation enhances onset dominance in sound localization

The Journal of the Acoustical Society of America 143, 786 (2018); 10.1121/1.5023221

Talkers produce more pronounced amplitude modulations when speaking in noise

The Journal of the Acoustical Society of America 143, EL121 (2018); 10.1121/1.5024404

Deep convolutional neural networks for estimating porous material parameters with ultrasound tomography The Journal of the Acoustical Society of America 143, 1148 (2018); 10.1121/1.5024341

Correspondence between sound propagation in discrete and continuous random media with application to forest acoustics

The Journal of the Acoustical Society of America 143, 1194 (2018); 10.1121/1.5024904 


\title{
Effects of flow gradients on directional radiation of human voice
}

\author{
Ville Pulkki, ${ }^{1, a)}$ Timo Lähivaara, ${ }^{2}$ and Ilkka Huhtakallio ${ }^{1}$ \\ ${ }^{1}$ Department of Signal Processing and Acoustics, Aalto University, Espoo, Finland \\ ${ }^{2}$ Department of Applied Physics, University of Eastern Finland, Kuopio, Finland
}

(Received 1 September 2017; revised 6 February 2018; accepted 6 February 2018; published online 23 February 2018)

In voice communication in windy outdoor conditions, complex velocity gradients appear in the flow field around the source, the receiver, and also in the atmosphere. It is commonly known that voice emanates stronger towards the downstream direction when compared with the upstream direction. In literature, the atmospheric effects are used to explain the stronger emanation in the downstream direction. This work shows that the wind also has an effect to the directivity of voice also favouring the downstream direction. The effect is addressed by measurements and simulations. Laboratory measurements are conducted by using a large pendulum with a loudspeaker mimicking the human head, whereas practical measurements utilizing the human voice are realized by placing a subject through the roof window of a moving car. The measurements and a simulation indicate congruent results in the speech frequency range: When the source faces the downstream direction, stronger radiation coinciding with the wind direction is observed, and when it faces the upstream direction, radiation is not affected notably. The simulated flow gradients show a wake region in the downstream direction, and the simulated acoustic field in the flow show that the region causes a wave-guide effect focusing the sound in the direction. (C) 2018 Acoustical Society of America. https://doi.org/10.1121/1.5025063

\section{INTRODUCTION}

It is commonly known that in windy conditions the voice of an utterer may be obscured by a listener in the upstream direction although heard clearly by a listener in the downstream direction at equal distances from the source. This might seem a simple phenomenon at first glance. However, if one studies in detail the acoustic communication chain in windy conditions, it is easy to notice that the moving medium may have an effect on many parts of the chain. The presence of the source, the receiver, and other objects and surfaces cause complex velocity gradients in the flow field. The movement of the medium might thus affect the sound generation in the source, the radiation from the source, the transmission through the atmosphere, and also the arrival of the sound at the listener.

In textbooks the effect causing the clearer hearing in the downstream direction is explained by refraction in the atmosphere. The wind speed is lower near the ground and increases with altitude causing a speed gradient in the flow field that bends the sound waves towards the ground in the downstream direction, and towards the zenith in the upstream direction. ${ }^{1}$ This effect, however, requires tens of meters of distance between the source and the listener to be prominent.

It is not generally known if the atmospheric effects are the only effects explaining the better audibility of voice in the downstream direction. It is an open question if the other parts of the acoustic communication chain are also affected by the movement of the medium, and if they are affected,

\footnotetext{
${ }^{\text {a)} E l e c t r o n i c ~ m a i l: ~ v i l l e . p u l k k i @ a a l t o . f i ~}$
}

does the effect contribute in the audibility of sound in the directions of up- and/or downstream. This work addresses the effect of wind to directional radiation from sources with similar dimensions with human source. The motivation for the study was that intuitively it seemed clear that the presence of a human body causes relatively strong velocity gradients in the flow field around the head, which might cause refractive effects in the emanation of sound waves from the mouth, whose effects to sound radiation were undocumented. This study shows that the wind also has an effect on directional radiation of voice favoring the downstream direction, which coincides with the common knowledge. The measurements are conducted first with a loudspeaker and second with a human subject in flow. Numerical simulations are conducted to further explain the mechanism causing the measured effects.

\section{BACKGROUND}

The human voice production system ${ }^{2}$ resembles a vibrating piston in a rigid sphere, or in a rigid cylinder, with diameter on the order of $15-30 \mathrm{~cm}$. The measured directional patterns of the human singing voice, ${ }^{3}$ and that of an artificial head $^{4}$ show that the human voice is almost omnidirectional at frequencies below approximately $1 \mathrm{kHz}$, radiating only a few decibels lower level to the rear hemisphere than to the frontal hemisphere. When the frequency exceeds $1 \mathrm{kHz}$, the response in the rear hemisphere drops in level and demonstrates complex directional lobe structures that depend on frequency; and at $4 \mathrm{kHz}$, the voice attenuation is approximately $10-20 \mathrm{~dB}$. The spectral energy in shouted speech sounds is mostly distributed to the frequency range of $0.3-3 \mathrm{kHz}$ with the most prominent peak between 1 and 
$2 \mathrm{kHz} .{ }^{5}$ The spectral peak thus exists in the region where the voice is notably directional, and also where the wavelengths of sound are of the same order with the diameter of the source.

The effect of flow on radiation from point-like sources has been studied theoretically. When a point-like source is placed into a medium with static flow, it radiates with higher level towards the upstream direction, and with lower level towards the downstream direction. ${ }^{6,7}$ In the upstream direction the pressure is amplified by a factor of $(1+v / c)^{2} /(1-v / c)^{2}$ when compared to the downstream direction, where $v$ is the speed of flow and $c$ is the speed of sound, and no effect is seen in the side directions. With a flow rate of $12 \mathrm{~m} / \mathrm{s}$, this means that sound should be amplified by $0.6 \mathrm{~dB}$ towards the upstream direction and attenuated by $0.6 \mathrm{~dB}$ towards the downstream direction. This effect is referred to as convective amplification, ${ }^{8}$ and it has been verified in measurements in ducts, ${ }^{9}$ with wind turbine blades, ${ }^{10}$ and with high-speed trains. ${ }^{11}$

When the human is in flow, complex patterns of flow gradients and turbulence are caused by the presence of the source. ${ }^{12}$ Such gradients have been studied in the context of microphone wind shields, and they are visualized for simplified geometries in Ref. 13. In Ref. 6 it is stated that the convective amplification effects may not hold with volumetric sources, especially at frequencies above the limit where the wavelength is of the same order with the dimensions of the source, although no further evidence or analysis is given. In the case of human source, the major radiation happens in the frequency region where the wavelength is of similar dimension with the source itself, and it can be assumed that the assumption of the point-like source is not valid generally with human voice.

A detailed overview of theories and their approximations of sound propagation in moving inhomogeneous media is provided in Ref. 14. However, the authors are not aware of theoretical- or measurement-based studies that would address the effect of flow gradients around a sound source similar to the human voice. A related field is the research of sound radiation from vehicles moving in air or water by using propellers or jets. A complex flow field is caused around the vehicles also in these cases, although the presence of the jet makes the flow field different in structure from the flow fields caused by wind around human subjects. Nevertheless, for example in the case of jet planes, the flow field caused by the vehicle has a strong effect on the directivity of the source. ${ }^{15}$

\section{MEASUREMENTS}

Ideally the effect of wind to voice should be measured with a real human subject in windy natural conditions. However, in natural conditions the flow of the air is disturbed by unpredictable gusts, and also the calibration of the measurement system is not possible since the wind cannot be stopped. The repeatable generation of flow would be possible in wind tunnels. However, the reflections from the walls of tunnels, prominent reverberation, and fan noise would make it difficult to extract the effect of flow to the radiation pattern.

In this work we chose to create the flow by moving the source with constant velocity through the air. Due to the relativity of motion, the air particles can be interpreted to move in relation with the source, which equates to a steady flow. An advantage of the method is the easy calibration of the system when the source is stationary. This method is commonly used in the audio industry for testing microphone wind shields. ${ }^{16,17}$ In the laboratory tests, a pendulum was used to move a deterministic sound source through the air (see Fig. 1), and in the practical measurements a car was used to move a real human shouter through the air (see Fig. 2).

\section{A. Loudspeaker in anechoic chamber in flow generated by a large pendulum}

A spherical loudspeaker with a diameter of $0.3 \mathrm{~m}$ was built for the purpose having similar dimensions with human head. Therefore, it is assumed to generate similar flow gradients around the source as is generated around the human head. Two different sources were utilized to produce sound. A simplified physical model of the vocal tract (tract source) simulates sound generation in voiced utterances such as vowels, and a source flush-mounted on the surface (piston source) simulates sound generation in unvoiced utterances with sound generation near the lips.

When the pendulum is released from upmost position, it accelerates rapidly the loudspeaker, and a stable speed is obtained for approximately $85 \mathrm{~ms}$ around the central position of the chamber, where the loudspeaker emits the measurement signal. Only the first swing of the pendulum is used in measurements to avoid uncontrollable effects in flow field due to previous swings. The instantaneous speed of the sources was computed from the measured angular speed of the pendulum. The speed of the tract source was on average $12.7 \mathrm{~m} / \mathrm{s}$ during the measurement window with a standard deviation of $0.08 \mathrm{~m} / \mathrm{s}$, and correspondingly $13.2 \mathrm{~m} / \mathrm{s}$ average

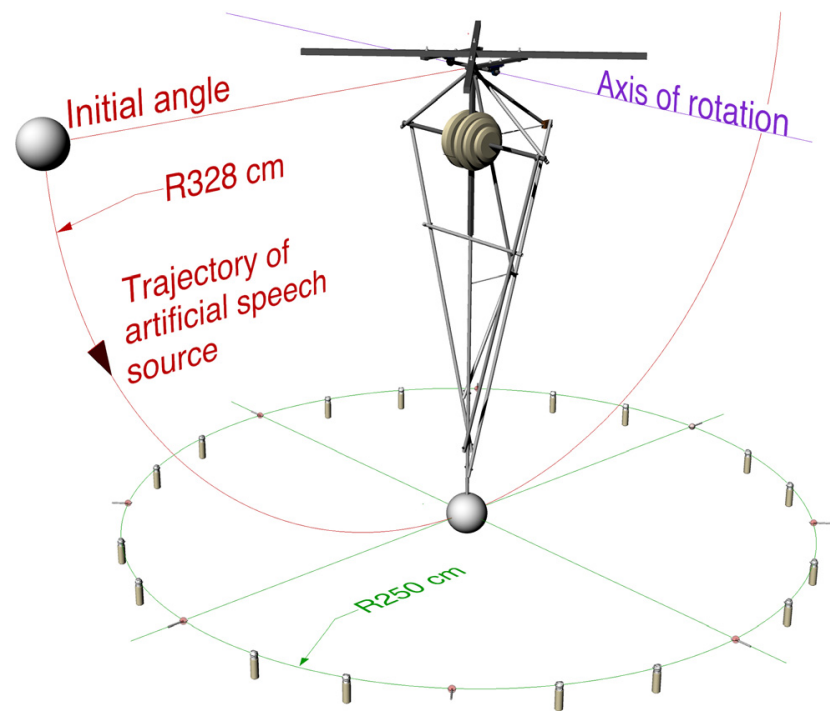

FIG. 1. (Color online) The pendulum used to create air flow around the source to measure the effect of $12.7-13.2 \mathrm{~m} / \mathrm{s}$ flow to directional pattern. The device was built inside a large anechoic chamber. 
with $0.13 \mathrm{~m} / \mathrm{s}$ standard deviation with the piston source. The loudspeaker moved for $108 \mathrm{~cm}$ and $19^{\circ}$ during this time period for the tract source, and the distance attenuation and frequency shift was compensated in the microphone signals. The processing thus compensates the relative movement of the loudspeaker and the microphones, and the result corresponds to the situation where the loudspeaker and the microphones are static with each other, and only the air is moving in between them.

The system can be considered to have the following advantages: the pendulum setup provides ideal flow relative to the source, as no other flow effects are present than those generated by the movement of the loudspeaker; the environment is also silent, and the source signal is deterministic, being a multitone; and the microphones are static with respect to the air, thus no noise or other effects of flow are present on response. The downsides of the system are that the measurement time is relatively short, that the path is slightly curved, and that the Doppler effect has to be cancelled.

\section{B. Real human voice source}

To investigate whether the controlled measurement results concurred with a real human source, a measurement with a subject was arranged. In the measurements, the subject was standing through the roof window of the car. The microphone setup was also built on top of the car, and two close-by microphones were used to estimate the signal radiated from the mouth. The car was driven back and forth on a taxi road of an airport on a calm day. The system is shown in Fig. 2.

The advantages of this method are that the source is real and the air flow around the subject corresponds realistically to natural windy conditions. However, there are a number of uncontrollable sources of error in this system, including: the signal is not deterministic and repeatable, the microphones are also positioned in air flow generating noise (potentially generating effects in directional patterns of them), the radiated signal is estimated with microphones outside of the mouth (the flow may affect the response from mouth to the close-by microphones), the flow turbulence causes background noise, and the external wind may affect the direction of flow. To partially overcome these problems, the measurements were repeated several times, and only the data with high signal-to-noise ratio were included in the results. The frequencies above $2 \mathrm{kHz}$ are not included in the plots, since the reference measurements with a small source revealed anomalies, which were clearly due to the effect of the flow on the directional patterns of the microphones. Furthermore, to emphasize the effect of flow on directional pattern of mouth in graphical representation of data, the omnidirectional average of radiation is normalized out from the responses.

\section{SIMULATIONS}

To verify the measurements, a computational simulation of the flow field and furthermore the acoustic field in the simulated flow field was conducted for the measurement setup in anechoic chamber. The advantages of this approach are that sound field can be directly monitored at any point, the geometry of the system and the flow field are known precisely, and also the simulated sound field can be visualized. The simulation was conducted by assuming a steady-state flow around the source, thus no time-varying changes in the flow field are not taken into account, and the simulation result has to be considered as a snap-shot of the flow-caused conditions. The disadvantage of the system is that it is not known if the computational model accurately simulates the immensely complex phenomena in sound field caused by temporally and spatially varying real flow field.

\section{A. Simulation of air flow}

In this work, the Ansys CFX5 software is used to compute the time-independent flow field (the steady-state problem). As the turbulence model, the shear-stress transport mode ${ }^{18}$ is used. In general, the transport model combines the robustness of the k- $\varepsilon$ model ${ }^{19}$ with the accuracy of the k$\omega$ model, ${ }^{20}$ making it applicable to a wide variety of turbulent flows. The problem geometry is a box $[0,1.5] \times[-1.5$, $1.5] \times[0,1.5] \mathrm{m}^{3}$ including the scatterer. The scatterer is placed at the origin and hence only one quarter of the total scatterer geometry is included in the model. Furthermore, the vocal tract is assumed to be parallel to the $y$-axis. The boundary conditions are chosen as follows: Scatterer geometry has the no-slip condition, planes $x=0 \mathrm{~m}$ and $z=0 \mathrm{~m}$ have the symmetry condition, planes $x=1.5 \mathrm{~m}$ and $z=1.5 \mathrm{~m}$ have the free slip condition, and finally boundaries $y=1.5 \mathrm{~m}$

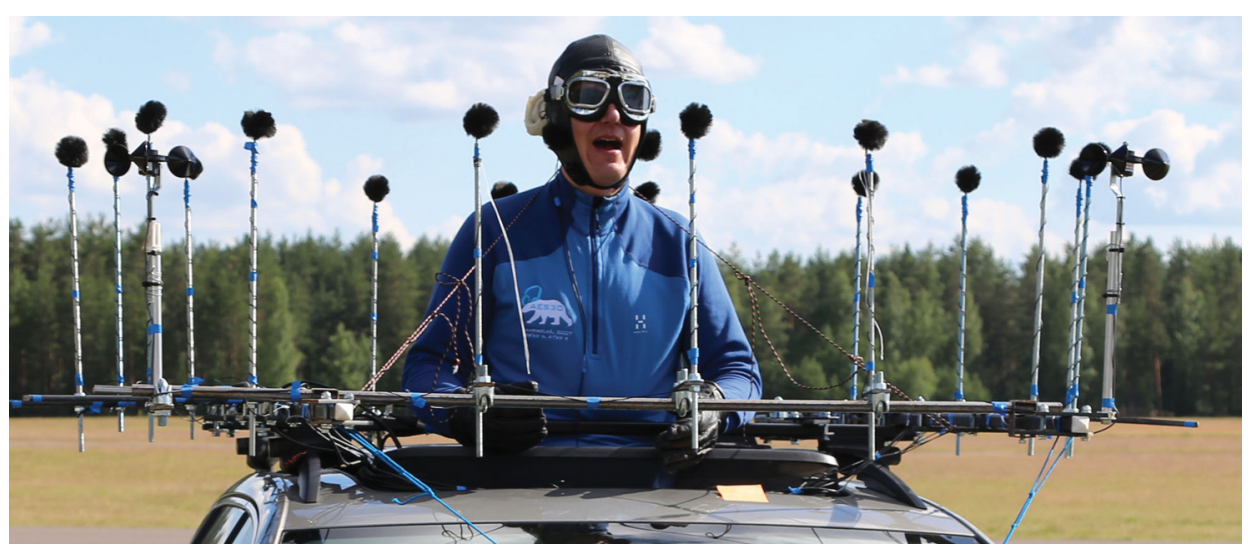

FIG. 2. (Color online) The system for measurements of the effect of flow to directional radiation from a real subject. 16 microphones and two wind speed meters surround the subject, two headmounted microphones capture the radiated signal, and the position of the head is kept constant via a head rest. $12 \mathrm{~m} / \mathrm{s}$ flow is generated by driving back and forth on a taxi road of an airport. 
and $y=-1.5 \mathrm{~m}$ have the outlet or inlet condition depending on the studied flow condition. The inlet velocity was $12 \mathrm{~m} / \mathrm{s}$.

For the computational grid used in the flow field calculations, the maximum element size was fixed to $1 \mathrm{~cm}$ inside the origin-centered $1.15 \mathrm{~m}$ radius circle. Furthermore, on the surface of the scatterer five inflation layers were used with a growth rate of 1.2. The maximum element size in other parts of the geometry was set to $25 \mathrm{~cm}$. As a material model, default parameters given to air at the temperature of $+25^{\circ} \mathrm{C}$ were used. Finally, as a convergence criteria for the iteration, a root-mean-square (RMS) value of $10^{-5}$ for the residual target was used.

\section{B. Acoustic simulation}

In this study, the discontinuous Galerkin (DG) finite element method, ${ }^{21}$ together with the explicit low-storage Runge-Kutta ${ }^{22}$ time stepping scheme was used as the simulation software for time-depended acoustic wave propagation (linearized Euler equations) in a non-uniform mean flow. The mean flow field was interpolated from the steady-state CFD simulation explained in Sec. IV A. In general, DG method has been applied to simulate acoustic waves in various studies. ${ }^{23-27}$ A detailed account of the method can be found in Ref. 21.

As a simulation geometry, a sphere with a radius of $1.15 \mathrm{~m}$ was used. Identically to the problem geometry used in the air flow simulations, the scatterer (loudspeaker) is also placed at the origin and the vocal tract is facing towards the positive $y$ axis. Propagation medium is air with speed of sound $346 \mathrm{~m} / \mathrm{s}$ and density $1.2 \mathrm{~kg} / \mathrm{m}^{3}$. As the boundary condition setup, the following was used: The sound source is introduced on the vibrating surface (at the end of the vocal tract) using the velocity inlet condition, while the other surfaces of the scatterer are modeled as a rigid wall. On the exterior surface, the outflow condition was used. In addition, a thin sponge zone was used to improve the outflow condition.

Acoustic simulations were performed on a computational grid having five tetrahedral elements per wavelength at the minimum. Furthermore, fifth-order basis functions together with zero initial condition were used in all simulations. As the computing infrastructure, a massively parallel processor supercomputer produced by Cray Inc. called $\mathrm{Sisu}^{28}$ (belonging to the XC40 family) was used.

\section{MEASUREMENT AND SIMULATION RESULTS}

\section{A. Simulation with point-like source in flow}

A reference simulation was conducted for a point-like sound source in a constant $12 \mathrm{~m} / \mathrm{s}$ flow in all positions of the field. The simulation result follows the convective amplification phenomenon, as in sound field an amplification of $0.6 \mathrm{~dB}$ in upstream direction and an attenuation of $0.6 \mathrm{~dB}$ in downstream direction resulted in, independent of frequency.

\section{B. Source oriented towards upstream}

The effect of flow on the radiation pattern is shown in Fig. 3. The radiation into the frontal hemisphere is either attenuated slightly or is not affected at all by the flow in pendulum measurements. In the rear of the source, some complex patterns can be observed with either positive or negative effect to the original directional pattern, which suggests that the flow affects the diffraction around the source in a notable way.

A deviation from the general trend is that at frequencies above $6 \mathrm{kHz}$, the tract source changes its radiation characteristics drastically. The frequency matches the wavelength where the first modes of the tract that are transversal to the main axis appear. It would appear that the flow affects the radiation differently for longitudinal modes and transversal modes of the tract.

The pendulum measurements with both source types produced very repetitive results and thus low $(<0.3 \mathrm{~dB})$ standard deviation in magnitude responses in the frontal hemisphere. Only in directions in the rear hemisphere of the source there are some slightly higher deviations $(\approx 0.4-$ $0.7 \mathrm{~dB}$ ), which is attributed to temporal instability of flow gradients affecting the diffracted sound differently at each measurement.

The measurements with the human source have to be considered less reliable than laboratory measurements due to many uncontrollable effects in the measurements. However, the average effect to radiation is minor and shows no clear trend with direction, and the measurement produced relatively low standard deviation $(<0.4 \mathrm{~dB})$ in most frequencydirection analysis positions.

The simulations shown in the right column of the figure show good agreement for the rear hemisphere of the source, where similar effects with two lobes can be observed than with the corresponding tract-source measurement. The results with frontal hemisphere differ somewhat from the measurement result. At frequencies between 1 and $2 \mathrm{kHz}$, an amplification of the magnitude response of about $0.3 \mathrm{~dB}$ was measured towards the front that is somewhat higher response than with measurement results, and somewhat smaller response than with the convective amplification phenomenon of $0.6 \mathrm{~dB}$ in this case. However, the effect vanishes at higher frequencies, where a relatively good match with measurements is again seen with the response of $-0.1 \mathrm{~dB}$.

To compare the results measured with different sources, a frequency average from the effect functions is measured with each source. The average is taken over the range of the highest spectral peak of the voice source, between 800 and $2500 \mathrm{~Hz}$, and they are shown in Fig. 4 for each source. To facilitate comparison, the average level has been scaled to $0 \mathrm{~dB}$ with each source. In general, the results from different sources are similar. Quite interestingly, the pendulum sources show an attenuation of about $0.5 \mathrm{~dB}$ in frontal directions and at most regions in the back directions an $0.5 \mathrm{~dB}$ amplification, whereas the human source and to the simulation results show no clear dependency on direction.

\section{Source oriented towards downstream}

The results from the measurements and simulations are shown in Fig. 5. Perhaps the most salient effect found in this work is that all cases show a clear beam towards the front direction of the source. With the pendulum and human voice 
Pendulum, tract
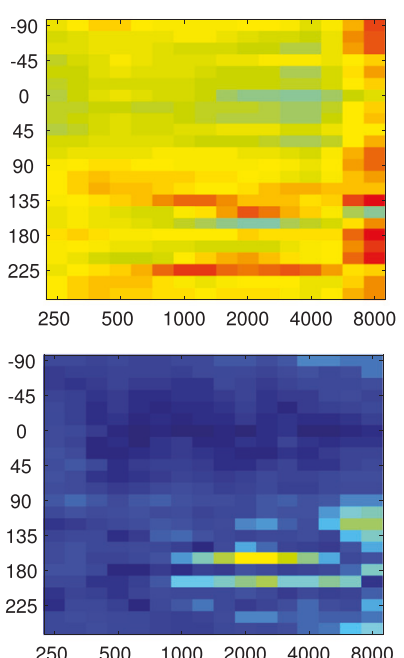

Pendulum, piston
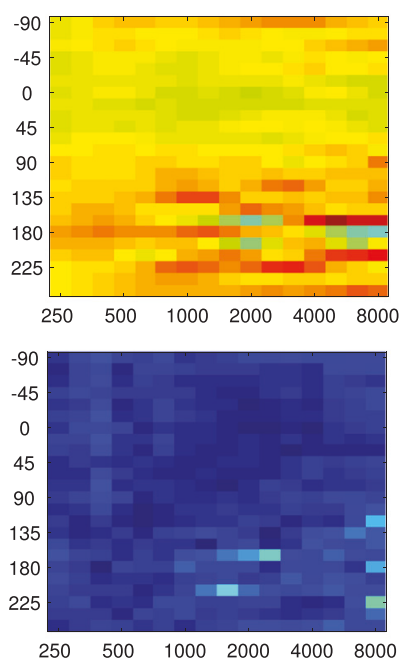

Translated, human
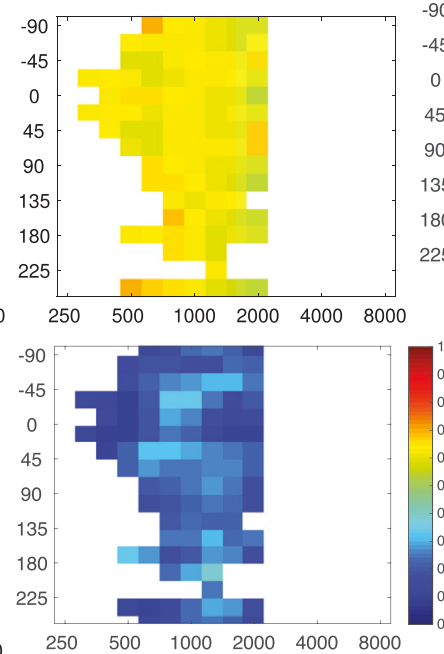

Simulation, tract
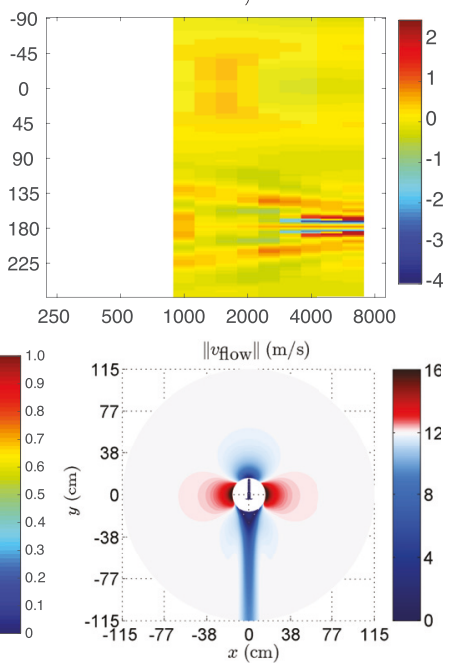

FIG. 3. Sound source facing upstream. Top row: the effect of flow on magnitude response at $1 / 3$-octave frequency bands ( $x$ axis) depending on azimuth angle ( $y$ axis), where $0^{\circ}$ is the orientation of the sound source. Bottom row: Corresponding standard deviations for measurements ( $n=3$ for pendulum, $n>24$ for human source). The approaches for measurements or simulation are tabulated above columns. On bottom right is shown the simulated speed of flow around spherical loudspeaker with tract source.

measurements the amplification ranges up to $1.5 \mathrm{~dB}$, and simulations even up to $4 \mathrm{~dB}$ in a very narrow beam.

The broader beam in the measurement results than in the simulation results is evidently caused by the fact that in the measurement the real flow field in the downstream direction is not temporally stable, which affects the direction of the beam and subsequently smears it in space. Such temporal instabilities of the flow field were not included in the simulation. The standard deviation of the results is still relatively low around the measured beams especially with pendulum measurements, which leads us believe that the beaming effect shown in the results is real. Even with human measurements the standard deviation is very low $(<0.3 \mathrm{~dB})$ directly in the front of the source at frequencies above $700 \mathrm{~Hz}$, where the beam is the most salient. Similarly with upstream-case measurements, complex effects in the rear hemisphere of the source are found, and the simulation also shows similar patterns.

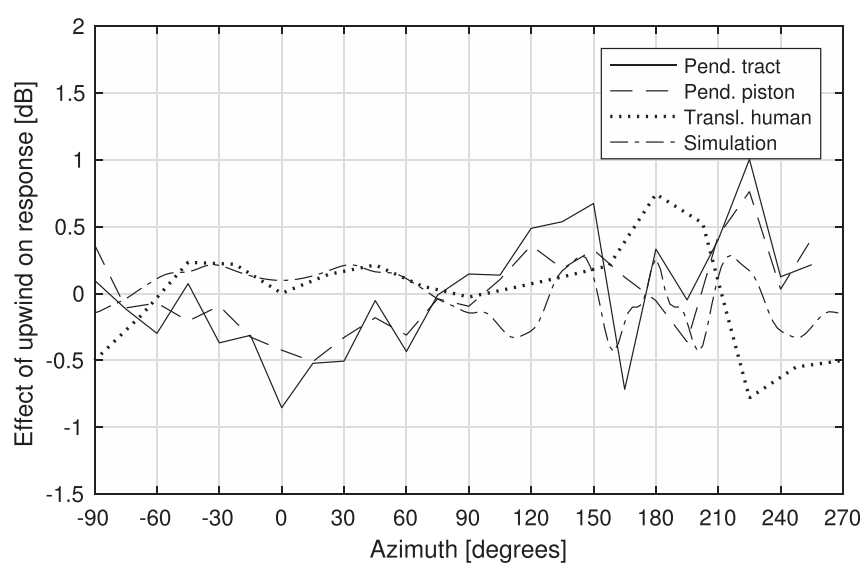

FIG. 4. The averaged effect of flow on upstream radiation on major speech frequencies. The results in Fig. 3 have been averaged over the frequency band, and the overall average of each source has been normalized to $0 \mathrm{~dB}$.
As with upstream-facing sources, a comparison of the wind-induced effects between each source is shown in Fig. 6 as averages over frequency band between 800 and $2500 \mathrm{~Hz}$. In general, the results from different sources are coherent. Each source type exhibits a similar lobe in the front, and in the rear hemisphere the sidelobes caused by the wind appear with the same directions and with similar amplitudes.

The simulated flow field in Fig. 5 can be used to explain the beaming effect. In this case the slower flow movement in the long wake region in the frontal direction of the source slows down the sound ray, as illustrated with the yellow arrow. The faster flow outside the wake region then bends the sound rays towards the front direction illustrated by the green arrow. The tubular region of wake therefore acts similarly as a wave guide. In the upstream case shown in Fig. 3, the tubular wake region does not have such a significant effect, since the source itself is not positioned inside the region.

The simulation also shows high flow speeds on the sides of the sources. This is the dominant region for sound diffraction towards the directions in the rear, and the large flow gradients evidently cause the changes in radiation to the rear directions.

\section{DISCUSSION}

The measurement results indicate that in windy conditions human voice radiates with higher efficiency towards the downstream when facing it. The existence of the effect with other sources than with human voice is now discussed. The cause of the effect is attributed to the slower sound velocities in the wake region and the baffle surrounding that creates a local waveguide for channeling the sound radiation. The authors speculate that the same effect may exist in other common situations found in the daily life, either in windy conditions or with moving sources. For example, when the wind flows around a chimney, or around a house in open space, a similar wake region is formed. The differences in 

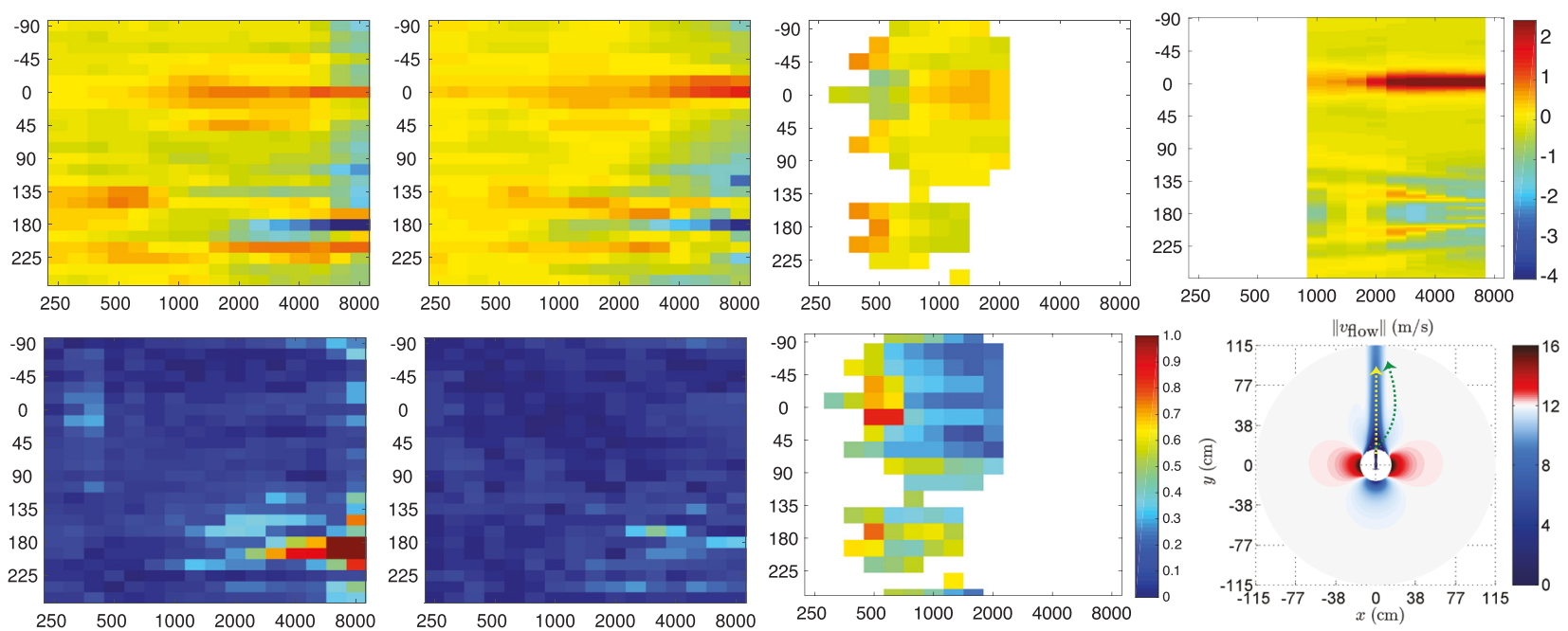

FIG. 5. As Fig. 3, but with sound source facing downstream.

sound speed caused by the wake region would potentially cause the sound emanated from the source to radiate with higher efficiency to the downstream direction. Another example of potential existence of the effect is with moving emergency vehicles equipped with a siren or a public address loudspeaker. The positioning of the sound source in relation to the wake region caused by the vehicle may affect the sound radiation from it.

The article thus adds some knowledge of the effects of wind on sound radiation from humans. It is noted that this work probably does not resolve totally the effects of wind to the communication channel from human voice to human listener in windy conditions. There may also exist other unknown phenomena. It is indicated in the results that the diffraction is affected by the flow, and also that the radiation from mouth is affected largely at frequencies where the transversal modes of the vocal tract appear. These phenomena are very likely to change the self-audition of the utterer, as their own voice may be perceived differently when facing upstream or downstream from the wind. Furthermore, the flow gradients around the distant listener may also affect his/hers perception,

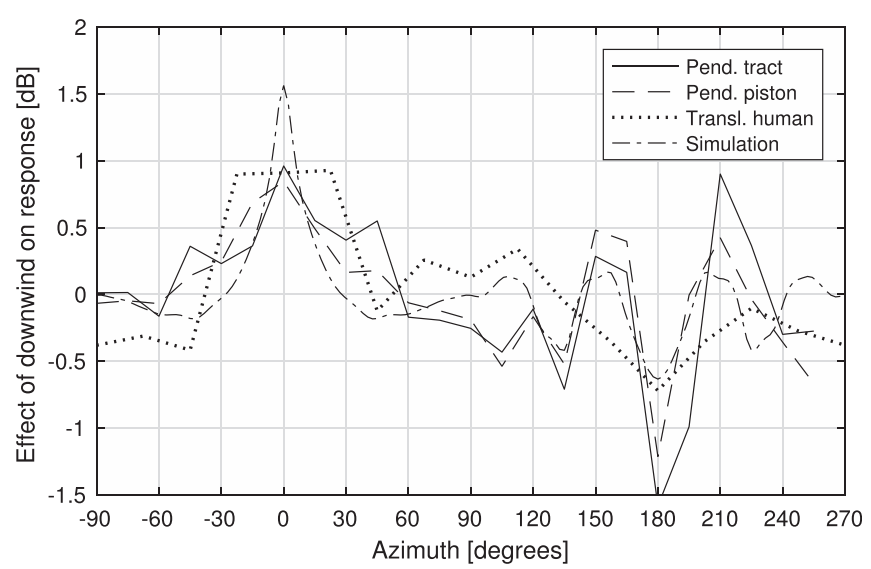

FIG. 6. The averaged effect of flow on downstream radiation on major speech frequencies. The results in Fig. 5 have been averaged over the frequency band, and the overall average of each source has been normalized to $0 \mathrm{~dB}$. as when the voice is radiated towards upstream, it will arrive at the listener from the direction of downstream. Therefore, the listener-caused flow gradients on the propagation path of sound may potentially cause lower reception of sound. These phenomena are left as subjects for future studies.

\section{CONCLUSION}

In windy conditions the presence of flow gradients caused by the human voice or by other sources with similar dimensions affects the sound radiation in such a way that sound is focused towards the downstream, provided that the source is facing it. This is explained by the focusing effect due to the flow gradients in the wake region generated by the sound source. This is observed with two different measurement approaches with different potential sources of error, and also in numerical simulations of ideal flow conditions.

It is concluded that the strong flow gradients near the surface of the source have an effect on how sound is diffracted around it. Strong direction-dependent effects on diffraction patterns are found in simulation results, which concur with the complex patterns found in corresponding measurements.

\section{ACKNOWLEDGMENTS}

The Academy of Finland has supported this work. The research leading to these results has received funding from the European Research Council under the European Community's Seventh Framework Programme (FP7/20072013)/ERC grant agreement No. [240453]. Alessandro Altoè and Kari Jääski did a great job driving the vehicles, and Leo McCormack helped with the English grammar.

\section{APPENDIX A: METHODS}

\section{Electroacoustic source in pendulum}

The measurements in the anechoic chamber were conducted with the setup shown in Fig. 1. The pendulum measured $328 \mathrm{~cm}$ from the pivot point to center of the source. An 
added mass of $70 \mathrm{~kg}$ was placed $60 \mathrm{~cm}$ down from the pivot point to adjust the mass distribution of the body to maximize the angular velocity. The pendulum was raised to its initial angle of $90^{\circ}$ by means of an electric chain hoist and permanent holding magnet with an electrical demagnetization coil. This allowed for a repeatable release of the pendulum. The angular rotation of the pendulum was monitored with a noncontact angle sensor.

In the case of the tract source, a moving coil cone driver (Eighteen Sound 3FE20) with $7 \mathrm{~cm}$ nominal diameter was coupled to a $17.5 \mathrm{~cm}$ long tube with diameter of $2.6 \mathrm{~cm}$. The other end of the tube was flush-mounted with the surface of a spherical baffle. For the piston source a Peerless 8309702 in. moving coil cone was flush-mounted directly to the baffle. The diameter of the sphere was $30 \mathrm{~cm}$ and it was made out of polycarbonate. The rear of the driver was enclosed in to an additional sealed volume inside the baffle.

A circular microphone array around the rest position of the pendulum was used to capture the directional response of the sources. The array consists of 24 microphones with $15^{\circ}$ spacing and $2.5 \mathrm{~m}$ radius as shown in Fig. 1. The microphones that were placed at directions of $0^{\circ}, 45^{\circ}, \ldots, 315^{\circ}$ were G.R.A.S 46AF $1 / 2$ in. free-field microphones and the remainder were Rode NT1-A large-diaphragm microphones with a cardioid pattern.

A multitone signal with 56 logarithmically spaced sine components between $100 \mathrm{~Hz}$ and $10 \mathrm{kHz}$ was generated. The reference response was measured by playing back the signal with the pendulum in the rest position, and after this the response in flow was recorded during the first swing of the pendulum. The pendulum was stopped before the start of each measurement. The sampling rate was $48 \mathrm{kHz}$, and an analysis window of 4096 samples around the time position corresponding to the lowest position of the pendulum were taken for analysis. The variation of distance at each time instance from the source to microphones was compensated by resampling and by compensating for the distance attenuation. The signal-to-noise ratio (SNR) of the recordings were measured by swinging the pendulum with and without sound generation. It was found, that at frequencies $\leq 2 \mathrm{kHz}$ the SNR is higher than $30 \mathrm{~dB}$ at all radiation directions, and with frequencies above $4 \mathrm{kHz}$ a slightly lower SNR of $20 \mathrm{~dB}$ is obtained in some cases, in the rear hemisphere of the source.

\section{Human voice in vehicle-generated wind}

The subject stood out of the roof window, with mouth elevated from the top of the car by approximately $45 \mathrm{~cm}$. A neck rest was used to keep the subject in constant distance from the microphones. A microphone array, two wind speed sensors, and the neck rest were mounted to a frame on top of the car. The microphones were positioned at the mouth level in directions $\left[0^{\circ}, 22.5^{\circ}, \ldots, 360^{\circ}\right]$ within distance of $90 \mathrm{~cm}$.

DPA 4060 lavaliere electret condenser microphones were used in the measurements, and they were covered first with foam windshields and second with furry Rycote windjammers to minimize noise caused by turbulence around the microphones. Two Inspeed Vortex cup anemometers were used to measure the speed of flow, and the driver adjusted the speed of the car according to the value of the frontal anemometer. The delays caused by different distances of the close and far microphones from the subject were compensated in the recorded signals.

The utterance to be used was selected to be /saana sahaa haapaa, siili viilaa tuиbaa, tuuli puhuu kiinaa, kissa kissa kissal, which lasted for 9-10 s. The C-weighted peak sound pressure level (SPL) produced by the subject were measured to vary between 85 and $90 \mathrm{~dB}$ in anechoic chamber at $1 \mathrm{~m}$ distance. The sentence was composed similarly as in Ref. 29 of words easy to pronounce, containing common vowels and consonants, and without any strong linguistical meanings.

The actual measurement was conducted in the following manner: The car was taken to the starting position of the 500 -m-long taxi way at Räyskälä airport in Finland; the utterance was recorded once with car still as reference measurement; the car was accelerated to the target velocity of $12 \mathrm{~m} / \mathrm{s}$ and the subject repeated the same sentence as many times until the driver signaled that he will start to decelerate; and the utterance was repeated again with car stopped as a second reference. The recordings with car stopped were used as reference in the analysis, where the natural wind speeds were measured to be in the range of $0.8-2.1 \mathrm{~m} / \mathrm{s}$. In the runs for upstream direction measurements, the measured instantaneous wind speed values averaged to value of $12.0 \mathrm{~m} / \mathrm{s}$ with standard deviation of $0.9 \mathrm{~m} / \mathrm{s}$. Correspondingly, in the runs for downstream direction measurements, the mean was $11.8 \mathrm{~m} / \mathrm{s}$ with standard deviation of $0.3 \mathrm{~m} / \mathrm{s}$. The day was half-cloudy without rain, and the temperature varied between $16^{\circ} \mathrm{C}$ and $22^{\circ} \mathrm{C}$.

\section{Computation of wind-induced change of directional patterns for human measurements}

A measured signal is denoted as $s(t)$. The multi-band signal $x(f, t)$, where $f$ is the center frequency, were filtered from $s(t)$ using cascaded third-order Butterworth high-pass and low-pass filters having cutoffs frequencies separated by a third octave. The instantaneous energy estimate of a narrowband signal is computed as

$$
y(f, t)=\mathrm{F}_{\mathrm{LP}}\left[x(f, t)^{2}\right],
$$

where operator $\mathrm{F}_{\mathrm{LP}}$ denotes first-order Butterworth low-pass filtering with cutoff frequency of $40 \mathrm{~Hz}$. This results in an estimation of the energy envelope of the microphone signal. It is assumed that the instantaneous energy is a summation of the instantaneous energies of the signal from the shouter and from the environmental noise:

$$
y(f, t)=y_{\text {signal }}(f, t)+y_{\text {noise }}(f, t) .
$$

From this the magnitude transfer function in octave bands from the source signal to far microphones is expressed as

$$
H(f)=\frac{\mathrm{E}\left[y_{\text {signal }}(f, t)\right]}{\mathrm{E}\left(y_{\text {source }}(f, t)\right)}=\frac{\mathrm{E}\left[y(f, t)-y_{\text {noise }}(f, t)\right]}{\mathrm{E}\left(y_{\text {source }}(f, t)\right)},
$$

where $\mathrm{E}$ denotes the expectation operator. 
With the human subject the variables $y_{\text {source }}(f, t)$ and $y_{\text {noise }}(t, f)$ are not known directly, and they have to be estimated. As the stimulus was natural speech, it contains both loud passages and pauses, and since the source microphone always delivers the signal with high SNR, the temporal positions of loud parts and pauses of the utterance can be estimated. $y(f, t)$ represents the instantaneous energy of the captured signal when the signal is present, and this is estimated best when the voice is on its loudest passages in a recorded utterance. Correspondingly, during the most silent passages in the utterance, the signal of the far microphone is

assumed to represent the background noise $y_{\text {noise }}(f, t)$. However, this requires that the levels of highest and lowest passages are separated well enough in level to enable reliable estimation of the transfer function. Therefore, the transfer function from the source microphone to the far microphones is estimated as

$$
\begin{aligned}
& A=\mathrm{E}_{\text {high20 }}\left[y(f, t), y_{\text {source }}(f, t)\right], \\
& B=\mathrm{E}_{\text {low } 10}\left(y(f, t), y_{\text {source }}(f, t)\right),
\end{aligned}
$$

where, $\mathrm{E}_{\text {low10 }}$ denotes an averaging process, where an average value is computed over the first signal variable, but only taking into account those samples occurring at the same time positions with the lowest $10 \%$ of sample values in the timesynchronous second signal variable. Correspondingly, $\mathrm{E}_{\text {high20 }}$ denotes averaging process for the first signal variable computed only over samples corresponding to the highest $20 \%$ of values in the second variable. The symbol $\emptyset$ denotes missing value. $A$ therefore estimates the average energy of signal with noise, and $B$ represents correspondingly only the noise. The condition $A \geq 8 B$ was set to discard all data that had lower estimated SNR ratio than $8 \mathrm{~dB}$ to ensure reliable estimation of the mean values.

$\hat{H}_{\text {flow }}(f, m)$ presents then the radiation pattern of the subject in flow, where $m$ is the index of the microphone in the setup. Correspondingly, $\hat{H}_{\text {calm }}(f, m)$ is the reference condition measured just after the run. The effect of flow to the radiation pattern is then estimated as

$$
\hat{H}_{\mathrm{w}}(f, m)=\frac{\hat{H}_{\text {flow }}(f, m)}{\hat{H}_{\text {calm }}(f, m)} .
$$

A large number of $\hat{H}_{\mathrm{w}}(f, m)$ values were computed from the results by comparing each $10 \mathrm{~s}$ utterance in flow to the reference measurement after the run. The mean and standard deviation were computed from the data for each far microphone and frequency band. If the number of missing values (symbol $\emptyset$ ) was larger than $10 \%$ in a case, the mean and standard deviation values were set to the value of $\emptyset$. The results were found to have some deviations in the level of sound radiated omnidirectionally, slightly different overall level was obtained for different center frequencies. As this study focuses the effect on directional pattern, the overall bias effect was removed from the plottings. The plots were normalized to values of $\hat{H}_{\mathrm{w}}(f, m) / \mathrm{E}_{\mathrm{m}}\left[\hat{H}_{\mathrm{w}}(f, m)\right]$, where $\mathrm{E}_{\mathrm{m}}$ denotes the average over microphones.

\section{Computation of wind-induced change of directional patterns for pendulum measurements}

The effect of flow to the transfer function from the source to the far-field microphones was estimated with the same method using instantaneous energy values in microphone signals, as in human-source measurements. However, $x_{\text {source }}$ is replaced by the electric multitone signal used to drive the loudspeaker. Furthermore, the level of background noise was estimated with the microphone signals recorded with realistic pendulum measurement swing, but without sound generation.

As the SNR was known to be adequate, instead of Eq. (A6) the response from the electrical signal to distant microphone signal was simply computed as

$$
\hat{H}(f, m)=\mathrm{E}\left[\frac{y(f, m, t)}{y_{\text {close }}(f, t)}\right],
$$

and Eq. (A7) was used to compute $\hat{H}_{\mathrm{w}}(f, m)$, which is presented without any further normalization.

${ }^{1} \mathrm{U}$. Ingård, "A review of the influence of meteorological conditions on sound propagation," J. Acoust. Soc. Am. 25(3), 405-411 (1953).

${ }^{2}$ J. L. Flanagan, Speech Analysis, Synthesis and Perception (Springer, New York, 1972).

${ }^{3}$ J. Pätynen and T. Lokki, "Directivities of symphony orchestra instruments," Acta Acust. united Acust. 96(1), 138-167 (2010).

${ }^{4}$ J. L. Flanagan, "Analog measurements of sound radiation from the mouth,” J. Acoust. Soc. Am. 32, 1613-1620 (1960).

${ }^{5} \mathrm{~W}$. O. Olsen, "Average speech levels and spectra in various speaking/listening conditions: A summary of the Pearson, Bennett, \& Fidell (1977) report," Am. J. Audiol. 7(2), 21-25 (1998).

${ }^{6} \mathrm{P}$. M. Morse and K. U. Ingard, Theoretical Acoustics (McGraw-Hill, New York, 1968).

${ }^{7}$ A. Dowling, "Convective amplification of real simple sources," J. Fluid Mech. 74(03), 529-546 (1976).

${ }^{8}$ A. Dowling, "Steady-state radiation from sources," in Handbook of Acoustics (Wiley, New York, 1998), pp. 99-117.

${ }^{9} \mathrm{U}$. Ingard and V. K. Singhal, "Upstream and downstream sound radiation into a moving fluid," J. Acoust. Soc. Am. 54(5), 1343-1346 (1973). 
${ }^{10}$ S. Oerlemans, P. Sijtsma, and B. M. López, "Location and quantification of noise sources on a wind turbine," J. Sound Vib. 299(4), 869-883 (2007).

${ }^{11} \mathrm{X}$. Zhang "The directivity of railway noise at different speeds," J. Sound Vib. 329(25), 5273-5288 (2010).

${ }^{12}$ J. D. Anderson, Jr., Fundamentals of Aerodynamics (McGraw-Hill Education, New York, 1985).

${ }^{13} \mathrm{Y}$. Xu and Z. Zheng, "A computational study of the effect of windscreen shape and flow resistivity on turbulent wind noise reduction," J. Acoust. Soc. Am. 129(4), 1740-1747 (2011).

${ }^{14} \mathrm{~V}$. E. Ostashev and D. K. Wilson, Acoustics in Moving Inhomogeneous Media (CRC, Boca Raton, FL, 2015).

${ }^{15} \mathrm{G}$ Gabard and R. J. Astley, "Theoretical model for sound radiation from annular jet pipes: Far-and near-field solutions," J. Fluid Mech. 549, 315-341 (2006).

${ }^{16}$ E. B. Brixen, "Microphones, high wind and rain," in Audio Engineering Society Convention, Audio Engineering Society (2005), p. 119.

${ }^{17} \mathrm{M}$. Brock, "Wind and turbulence noise of turbulence screen, nose cone and sound intensity probe with wind screen," Brüel \& Kjær Tech. Rev. 4, 32-39 (1986).

${ }^{18} \mathrm{~F}$. Menter, "Two-equation eddy-viscosity turbulence models for engineering applications," AIAA J. 32(8), 1598-1605 (1994).

${ }^{19} \mathrm{~W}$. Jones and B. Launder, "The prediction of laminarization with a twoequation model of turbulence," Int. J. Heat. Mass. Transf. 15, 301-314 (1972).

${ }^{20} \mathrm{D}$. Wilcox, Turbulence Modeling for CFD (DCW Industries Inc., La Canada, CA, 1993)
${ }^{21}$ J. Hesthaven and T. Warburton, Nodal Discontinuous Galerkin Methods: Algorithms, Analysis, and Applications (Springer, New York, 2007).

${ }^{22}$ M. Carpenter and C. Kennedy, "Fourth-order $2 \mathrm{~N}$-storage Runge-Kutta schemes," Technical Report (NASA-TM-109112, 1994).

${ }^{23}$ B. Cockburn, G. Karniadakis, and C.-W. Shu, eds., Discontinuous Galerkin Methods Theory, Computation and Applications (SpringerVerlag, Berlin Heidelberg, 2000).

${ }^{24}$ P. Delorme, P. Mazet, C. Peyret, and Y. Ventribout, "Computational aeroacoustics applications based on a discontinuous Galerkin method," C. R. Méc. 333(9), 676-682 (2005).

${ }^{25} \mathrm{M}$. Bernacki, S. Lanteri, and S. Piperno, "Time-domain parallel simulation of heterogeneous wave propagation on unstructured grids using explicit, nondiffusive, discontinuous Galerkin methods," J. Comput. Acoust. 14(01), 57-81 (2006).

${ }^{26} \mathrm{~T}$. Lähivaara and T. Huttunen, "A non-uniform basis order for the discontinuous Galerkin method of the 3D dissipative wave equation with perfectly matched layer," J. Comput. Phys. 229, 5144-5160 (2010).

${ }^{27}$ A. Modave, A. St-Cyr, and T. Warburton, "GPU performance analysis of a nodal discontinuous Galerkin method for acoustic and elastic models," Comput. Geosci. 91, 64-76 (2016).

${ }^{28}$ Sisu supercomputer, "CSC-IT Center for Science Ltd.," https:// research.csc.fi/sisu-supercomputer (2017).

${ }^{29}$ J. Pohjalainen, T. Raitio, S. Yrttiaho, and P. Alku, "Detection of shouted speech in noise: Human and machine," J. Acoust. Soc. Am. 133(4), 2377-2389 (2013). 